\title{
Les stratégies éducatives du personnel en milieu de garde avec les enfants présentant des troubles du comportement
} Appréciation des effets d'un programme

\section{Educative Strategies of Day-Care Personnel with Children Who} Have Behavioural Problems Appreciation of the Effects of the Program

Las estrategias educativas del personal en medio de guarda con niños que presentan problemas de comportamiento Apreciación de las repercusiones de un programa

\author{
Daniel Turcotte, Marie-Christine Saint-Jacques, Annick St-Amand et Émilie \\ Dionne
}

Volume 33, numéro 2, automne 2005

Nouvelles tendances à l'égard de la petite enfance

URI : https://id.erudit.org/iderudit/1079106ar

DOI : https://doi.org/10.7202/1079106ar

Aller au sommaire du numéro

\section{Éditeur(s)}

Association canadienne d'éducation de langue française

\section{ISSN}

0849-1089 (imprimé)

1916-8659 (numérique)

Découvrir la revue

\section{Citer cet article}

Turcotte, D., Saint-Jacques, M.-C., St-Amand, A. \& Dionne, É. (2005). Les stratégies éducatives du personnel en milieu de garde avec les enfants présentant des troubles du comportement : appréciation des effets d'un programme. Éducation et francophonie, 33(2), 161-181.

https://doi.org/10.7202/1079106ar

\section{Résumé de l'article}

Les problèmes de comportement chez les enfants doivent faire l'objet d'une attention particulière en raison des difficultés d'adaptation qui guettent ces derniers à moyen et à long termes. La présence de ces problèmes en bas âge laisse présager d'éventuelles difficultés d'adaptation telles que l'abandon scolaire, la toxicomanie, le rejet de la part des camarades, les difficultés interpersonnelles et les conduites délinquantes. Pour contrer cette trajectoire développementale problématique, il importe d'intervenir précocement dans la vie de l'enfant. Or, l'intervention auprès des enfants qui ont des problèmes de comportement constitue un défi majeur pour le personnel en milieu de garde. Cet article porte sur un programme d'intervention qui est offert au personnel des milieux de garde pour les aider à composer avec ce type de situations. Les données, recueillies dans le cadre d’une démarche qualitative, révèlent que les participantes attribuent des effets positifs au programme. En faisant une meilleure analyse des besoins que l'enfant exprime par ses comportements, elles sont en mesure d'opter pour des stratégies d'intervention mieux adaptées et plus efficaces. Cependant, elles soulignent qu'une intervention efficace auprès d'un enfant en difficulté exige une action concertée des principaux acteurs qui l'entourent, d'où l'importance d'associer les parents à la démarche d'intervention.
Tous droits réservés (C) Association canadienne d'éducation de langue française, 2005
Cedocument est protégé par la loi sur le droit d'auteur. L'utilisation des services d'Érudit (y compris la reproduction) est assujettie à sa politique d'utilisation que vous pouvez consulter en ligne.

https://apropos.erudit.org/fr/usagers/politique-dutilisation/ 


\section{Les stratégies éducatives du personnel en milieu de garde avec les enfants présentant des troubles du comportement : appréciation des effets d'un programme}

\section{Daniel TURCOTTE}

Université Laval, Québec, Québec, Canada

Marie-Christine SAINT-JACQUES

Université Laval, Québec, Québec, Canada

Annick ST-AMAND

Université Laval, Québec, Québec, Canada

Émilie DIONNE

Université Laval, Québec, Québec, Canada

\section{RÉSUMÉ}

Les problèmes de comportement chez les enfants doivent faire l'objet d'une attention particulière en raison des difficultés d'adaptation qui guettent ces derniers à moyen et à long termes. La présence de ces problèmes en bas âge laisse présager 
d'éventuelles difficultés d'adaptation telles que l'abandon scolaire, la toxicomanie, le rejet de la part des camarades, les difficultés interpersonnelles et les conduites délinquantes. Pour contrer cette trajectoire développementale problématique, il importe d'intervenir précocement dans la vie de l'enfant. Or, l'intervention auprès des enfants qui ont des problèmes de comportement constitue un défi majeur pour le personnel en milieu de garde. Cet article porte sur un programme d'intervention qui est offert au personnel des milieux de garde pour les aider à composer avec ce type de situations. Les données, recueillies dans le cadre d'une démarche qualitative, révèlent que les participantes attribuent des effets positifs au programme. En faisant une meilleure analyse des besoins que l'enfant exprime par ses comportements, elles sont en mesure d'opter pour des stratégies d'intervention mieux adaptées et plus efficaces. Cependant, elles soulignent qu'une intervention efficace auprès d'un enfant en difficulté exige une action concertée des principaux acteurs qui l'entourent, d'où l'importance d'associer les parents à la démarche d'intervention.

\section{ABSTRACT}

\section{Educative Strategies of Day-Care Personnel with Children Who Have Behavioural Problems: Appreciation of the Effects of the Program}

Daniel Turcotte, Marie-Christine Saint-Jacques, Annick St-Amand, and Émilie Dionne

Research Centre on the Adaptation of Youth and Families at Risk

Université Laval, Québec

It is important to pay particular attention to children's behaviour problems because of the adaptation difficulties these children may face over the medium and long term. If children have these problems at an early age, they may be at risk of adaptation problems, such as dropping out of school, drug addiction, being rejected by other young people, interpersonal problems, and delinquent behaviour. Early intervention is important in counteracting this problematic development trajectory. Intervention with children who have behaviour problems is a major challenge for day-care personnel. This article is based on an intervention program offered to daycare personnel to help them deal with these types of situations. The data, gathered during a qualitative process, reveals that participants find that the program has positive effects. By doing a better analysis of the needs that children express through these behaviours, they are able to choose better adapted and more effective intervention strategies. However, they emphasize that effective intervention with a child in difficulty requires the concerted action of everyone working with the child, thus the importance of working with the parents during the intervention process. 


\section{RESUMEN}

\section{Las estrategias educativas del personal en medio de guarda con niños que presentan problemas de comportamiento : apreciación de las repercusiones de un programa.}

Daniel Turcotte, Marie-Christine Saint-Jacques, Annick St-Amand y Émilie Dionne Centro de investigaciones sobre la adaptación de los niños y las familias frágiles Universidad Laval, Quebec

Los problemas de comportamiento de los niños deben ser tratados con una atención muy particular a causa de las dificultades de adaptación que pesan sobre ellos a mediano y largo plazo. La presencia de dichos problemas en edad temprana permite pronosticar eventuales dificultades de adaptación como el abandono escolar, la toxicomanía, el rechazo de la parte de los camaradas, las dificultades interpersonales y los comportamientos delincuentes. Para confrontar esta trayectoria evolutiva problemática, es importante intervenir de manera precoz en la vida de los niños. Ahora bien, la intervención entre los niños que presentan problemas de comportamiento constituye un desafío de talla para las empleadas de las guarderías. Este artículo presenta un programa de intervención que se ofrece al personal de las guarderías para ayudarlos a transigir con ese tipo de situaciones. Los datos, recogidos en el cuadro de un enfoque cualitativo, muestran que las participantes atribuyen efectos positivos al programa. Al realizar un análisis más adecuado de las necesidades que los niños expresan a través sus comportamientos, ellas pueden optar por estrategias de intervención más adecuadas y eficaces. Sin embargo, ellas recalcan que una intervención eficaz entre los niños con problemas exige una acción concertada entre los actores principales que rodean al niño, de ahí la importancia de asociar los padres de familia a la intervención.

\section{Introduction}

Il est largement reconnu que les problèmes de comportement chez les enfants doivent faire l'objet d'une attention particulière en raison des difficultés d'adaptation qui les guettent à moyen et à long termes. La présence de ces problèmes en bas âge laisse présager d'éventuelles difficultés d'adaptation telles que l'abandon scolaire, la toxicomanie, le rejet, les difficultés interpersonnelles, les conduites délinquantes, la criminalité, les problèmes de santé, etc. (Kazdin, 1987; Kratzer et Hodgins, 1997; Lacourse, Côté, Nagin, Vitaro, Brendgen et Tremblay, 2002; Vitaro, Dobkin, Gagnon et Leblanc, 1994; Werner et Smith, 1989).

Bien qu'il soit difficile de statuer sur les causes des problèmes de comportement, puisqu'ils peuvent résulter à la fois des caractéristiques de l'enfant, des dysfonctions 
familiales et de l'interaction des deux, tous en sont affectés. Ainsi, l'enfant subit des conséquences de cette situation par les réactions négatives qu'il provoque. En outre, les problèmes de comportement ont des répercussions négatives sur les différents milieux de vie dans lesquels évolue l'enfant. La famille est évidemment la plus directement touchée par cette problématique. Devant leurs difficultés à contrôler les comportements négatifs de leur enfant, les parents se culpabilisent, s'épuisent et en viennent à se sentir totalement impuissants. La plupart du temps, l'inefficacité de leurs efforts engendre une dynamique d'interactions négatives "parents-enfants" qui contribue à exacerber le problème. C'est alors toute la dynamique familiale qui s'en trouve ébranlée de fait du climat de tension qui se développe au sein de la fratrie (Webster-Stratton et Herbert, 1995).

Le milieu de garde est un autre contexte de vie particulièrement affecté par les problèmes de comportement de l'enfant. Ces comportements nuisent au climat du milieu de garde et entravent le bon déroulement des activités. Qu'ils soient témoins ou victimes de gestes agressifs, les autres enfants du groupe réagissent à ces comportements. Certains ont des réactions d'insécurité, d'anxiété ou de perte de contrôle qui exigent plus d'attention de la part des adultes (Kaiser et Sklar Rasminsky, 1999); d'autres cherchent plutôt à imiter leurs pairs turbulents et copient les comportements problématiques. Dans de telles situations, les programmations sont souvent perturbées puisque le personnel doit passer beaucoup de temps à gérer les crises, à consoler les enfants et à contrôler les comportements inadéquats. L'étude de Coutu, Lavigueur, Dubeau et Tardif (2003) indique que les comportements de colère et d'agressivité physique sont les situations les plus difficiles à gérer pour les éducatrices. On conçoit aisément que la présence, au sein d'un milieu de garde, d'un enfant qui présente des problèmes de comportement puisse être lourde de conséquences à la fois pour l'enfant, pour ceux qui l'entourent et pour les membres du personnel. Chez ces derniers, il peut en résulter un sentiment d'incompétence, une impression d'inadéquation, des remords, des doutes, voire même une remise en question du choix de la profession (Kaiser et Sklar Rasminsky, 1999).

Afin de contrer cette trajectoire développementale problématique, il importe d'intervenir le plus précocement dans la vie de l'enfant pour éviter que se cristallisent les comportements perturbateurs. Toutefois, le personnel en milieu de garde se sent démuni à l'égard de ces comportements. En effet, selon certaines études, l'intervention auprès des enfants ayant des problèmes d'agressivité constitue le thème de formation le plus souhaité par le personnel en milieu de garde (Coutu et coll., 2003). Cet article porte sur un programme d'intervention (le Service d'aide à l'enfant et son milieu - SAEM) s'adressant au personnel des milieux de garde pour l'aider à composer avec un (ou des) enfant présentant des problèmes de comportement. Ce programme s'appuie sur une évaluation précise des problèmes de comportement de l'enfant et sur le développement de stratégies visant à répondre aux besoins que les comportements de l'enfant traduisent. La présente étude vise, d'une part, à déterminer les stratégies éducatives utilisées par le personnel en milieu de garde à l'égard des problèmes de comportement des enfants et, d'autre part, à examiner l'utilité du programme selon l'appréciation formulée par les participantes. 
Les stratégies éducatives du personnel en milieu de garde avec les enfants présentant des troubles du comportement: appréciation des effets d'un programme

Le texte se divise en quatre parties principales. La première présente un survol rapide des programmes qui peuvent soutenir l'action du personnel éducatif. Les deux suivantes décrivent respectivement la nature du programme SAEM et la méthode de recherche utilisée pour en cerner la portée. La dernière partie porte sur les stratégies éducatives utilisées en milieu de garde et sur l'appréciation du programme par les participantes.

\section{La prévention de l'inadaptation sociale de l'enfant}

Au cours des quarante dernières années, de nombreux programmes préventifs ont été développés à l'intention des enfants d'âge préscolaire, et particulièrement ceux des milieux défavorisés. Qu'il s'agisse des programmes comme Head Start, Abecederian Carolina Program ou Fast Track aux États-Unis ou des programmes Apprenti-Sage, 1,2,3 Go ! ou Fluppy au Québec (CCPRG, 1997; Piché, Roy et Couture, 1992; Bastien, Plante et Cotte, 1995; Capuano, 1995; Weisberg et Greenberg, 1998; Vitaro et Gagnon, 2000). Également, en milieu scolaire, plusieurs programmes de prévention et d'intervention précoce ont été mis sur pied à l'intention des élèves présentant des problèmes d'adaptation : programmes d'entraînement aux stratégies de résolution de problèmes, programmes d'intervention impliquant des pairs, programmes d'entraînement aux habiletés sociales, etc. Ces programmes visent notamment à stimuler le développement affectif et social des jeunes, à développer leurs stratégies de gestion des conflits, à les amener à acquérir des habiletés sociales et à mieux gérer leur stress. La philosophie de ces programmes, leurs cibles d'intervention (jeunes, parents, éducateurs, professeurs, etc.), les instruments utilisés pour mesurer leurs impacts et leurs impacts eux-mêmes sont très diversifiés (Desbiens, 2000), d'où la difficulté d'en tracer un portrait comparatif précis. Fortin et Bigras (1997), suite à une recension de programmes préventifs destinés aux enfants à risque, mentionnent que si le degré d'optimisme quant à l'efficacité de tels programmes varie énormément, il est très difficile d'en établir avec précision l'efficacité

Les programmes de prévention des problèmes d'adaptation sociale des enfants conçus et adaptés spécifiquement pour les milieux de garde à l'enfance sont relativement peu nombreux. en raison des limites méthodologiques imposées par le contexte d'évaluation de ces programmes, notamment, la difficulté d'avoir un groupe contrôle, le nombre de sujets peu élevé, les taux d'abandon élevés et la variabilité dans l'application des programmes.

Par ailleurs, les programmes de prévention des problèmes d'adaptation sociale des enfants conçus et adaptés spécifiquement pour les milieux de garde à l'enfance sont relativement peu nombreux (Coutu, Lavigueur, Dubeau et Harvey, 1995; Hamel, 1995; MSSSQ, 1997; Reese, Vera, Simon et Ikeda, 2002). Or, ces milieux sont des lieux stratégiques pour la prévention des problèmes d'adaptation sociale. En effet, selon les données de Statistiques Canada (2005), plus de $60 \%$ des enfants âgés de six mois à cinq ans au Québec sont confiés à un mode de garde quelconque. En outre, plusieurs enfants fréquentent leur milieu de garde pour une période relativement longue, ce qui les amène à y développer des liens importants. Donc, plusieurs facteurs «militent fortement en faveur du développement et de l'intégration d'activités 
de prévention des problèmes d'adaptation sociale des enfants dans les milieux de garde à l'enfance» (Coutu et coll., $2003:$ 7).

Non seulement les programmes sont-ils peu nombreux, mais il existe peu de données concernant les stratégies éducatives des éducatrices qui interviennent auprès des enfants, notamment ceux qui présentent des difficultés d'adaptation. Or, il s'agit là d'un volet majeur de l'intervention auprès des enfants en difficulté, car ce sont ces personnes qui interviennent au quotidien auprès d'eux. D'ailleurs, PoliquinVerville et Royer (1992) soulignent que l'évaluation de programmes devrait tout autant porter sur les stratégies éducatives du personnel que sur les progrès du jeune.

\section{Les stratégies éducatives en milieu de garde}

Les stratégies éducatives sont des techniques d'intervention concrètes destinées aux jeunes; il s'agit d'actions concrètes et de moyens pratiques utilisés par le personnel éducateur dans ses interventions auprès des enfants et des jeunes. Ces stratégies visent à contrôler ou à développer les comportements en conformité avec les attentes des adultes envers l'enfant (Grosenick et coll., 1985).

Certains auteurs distinguent deux grandes catégories de stratégies éducatives : les interventions directes et indirectes (Ministère de la Famille et de l'Enfance, 1998). Les interventions directes incluent des interventions telles qu'ignorer les comportements agaçants, faire preuve d'humour pour dédramatiser une situation, offrir des moments privilégiés d'attention, intervenir lorsque survient un comportement dangereux ou injuste ou façonner les comportements souhaités par la discussion ou le renforcement positif. Les interventions indirectes visent à modifier certains comportements problématiques en agissant sur des éléments extérieurs à l'enfant. L'aménagement des locaux, la mise en place d'un espace d'intimité, l'adoption d'un programme d'activités variées et adaptées, la communication avec les parents sont autant d'exemples d'interventions.

Pour sa part, Essa (2002) propose une typologie plus détaillée. Elle distingue dix techniques de modification des comportements. Certaines favorisent le maintien des comportements positifs, alors que d'autres visent plutôt la diminution des comportements perturbateurs. Ces dix techniques sont: 1) le renforcement, 2) l'ignorance, 3) l'isolement de l'enfant, 4) le retrait volontaire, 5) la prévention, 6) la réorientation, 7) la discussion, 8) la créativité dans la solution de problèmes, 9) la période spéciale et 10) le tableau de renforcement.

Selon Essa (2002), il est primordial que l'adulte sache comment faire face adéquatement aux comportements inacceptables des jeunes. Sa réaction est susceptible d'entraîner une foule de répercussions, car l'image que l'enfant a de lui-même se construit principalement à partir des réactions de l'adulte à ses comportements. Lorsque cette réaction a lieu en milieu de garde, elle peut avoir un impact non seulement sur l'enfant, mais sur l'ensemble du groupe. Elle peut tout aussi bien contribuer au maintien d'un climat positif qu'entraver le déroulement des activités. Les techniques proposées par Essa (2002) fournissent des pistes concrètes pour guider l'adulte dans le choix de ses réactions face aux comportements d'un enfant. Mais il doit garder en tête que chaque cas est unique. Donc, il doit utiliser son jugement afin 
de choisir les bonnes techniques en fonction de l'enfant, du contexte et de la nature du comportement en cause. D'où l'importance de faire une analyse approfondie de chaque situation. C'est là l'idée centrale sur laquelle s'appuie le Programme SAEM.

\section{Description du programme SAEM}

Le Programme SAEM (Service d'aide à l'enfant et son milieu) est un programme préventif destiné aux enfants âgés de 0 à 5 ans qui présentent des problèmes de comportement. Le programme comporte trois volets qui peuvent être mis en lien avec les types de prévention distingués par l'Institute of Medecine (1994 cité dans Coutu et coll., 2003). Le premier volet a un caractère universel, car il s'adresse à l'ensemble des parents; il s'agit d'activités de formation aux parents. Le second est sélectif et prend la forme de groupes pour familles défavorisées. Le troisième vise spécifiquement les enfants qui présentent des problèmes de comportement; il s'agit du volet Aide et soutien aux parents et au personnel en milieu de garde. C'est ce dernier volet qui constitue l'objet de la présente étude.

Dans ce volet, l'intervention s'appuie sur le postulat que les enfants expriment des besoins particuliers par leurs comportements inadéquats. Le processus consiste essentiellement à cerner ces besoins et à apporter une aide et un soutien concrets aux parents et au personnel en milieu de garde pour qu'ils puissent fournir une réponse adéquate aux besoins de l'enfant. Différents principes orientent l'intervention : action à court terme dans les milieux de vie de l'enfant, approche d'enseignement et de «coaching", recherche de solutions concrètes dans "l'ici et maintenant", valorisation des forces des différents acteurs, reconnaissance des besoins de l'enfant, encouragement à l'autonomie et interdisciplinarité (Bouchard, Girouard et St-Amand, 2004; Paradis et Cantin, 1993).

Les demandes de service peuvent provenir soit des parents, soit du milieu de garde. Dans la plupart des cas, il est privilégié que les interventions se fassent à la fois avec les parents et avec le milieu de garde, mais l'absence de l'un ou l'autre des parties n'empêche pas le processus de se mettre en branle. Globalement, l'intervention s'effectue en trois étapes : 1) évaluation de la situation problématique, 2) élaboration d'un plan d'intervention, 3) application du plan avec une évaluation continue des résultats. Le processus d'intervention se déroule sur trois à six rencontres, structurées en fonction des étapes du processus, qui ont lieu dans les milieux de vie de l'enfant, soit la famille et le milieu de garde, et qui se déroulent à un rythme variable selon les besoins et le contexte.

\section{La méthode d'évaluation}

Pour évaluer l'utilité de ce programme, un devis basé sur une approche qualitative a été privilégié. Étant donné qu'il s'agissait d'une première évaluation de ce programme et considérant les défis d'une collecte d'information auprès d'éducatrices 
qui sont en présence d'enfants, nous avons opté pour cette approche parce qu'elle facilite la prise en compte des éléments contextuels, qu'elle permet de saisir l'expérience dans la perspective de ceux qui la vivent et qu'elle autorise plus de souplesse quant à la procédure de collecte de données (Deslauriers et Kérésit, 1997; Marshall et Rossman, 1999; Patton, 1987). Deux types de données ont été utilisés pour cerner l'influence du programme sur la gestion des problèmes de comportement. D'une part, les stratégies éducatives utilisées pas les éducatrices ont été documentées à deux moments, soit avant et après la mise en place du programme. D'autre part, une mesure d'appréciation portant sur la satisfaction et sur les effets perçus a été effectuée à la fin du programme.

Les données ont été collectées par entrevue avant que l'intervention débute (temps 1) et au moment où l'intervention prenait fin (temps 2) auprès d'éducatrices qui ont fait appel au programme SAEM entre décembre 2001 et mars 2003. Au temps 1 , les éducatrices $(n=41)$ ont été rencontrées en personne, le plus souvent dans leur milieu de travail. Les données ont été collectées à partir d'un schéma d'entrevue à questions ouvertes dont le contenu portait sur les problèmes de comportement de l'enfant et sur les stratégies de l'éducatrice à son endroit, avant le début de l'intervention. Les problèmes de comportement étaient explorés en demandant à l'éducatrice de "décrire brièvement ce qui est plus problématique chez l'enfant" et en lui faisant préciser les impacts de ces comportements sur elle, sur le groupe, sur les autres enfants. Pour cerner les stratégies utilisées, la question suivante était posée: "Jusqu'à maintenant, quels moyens concrets avez-vous mis en place en réaction aux comportements décrits précédemment?». L'éducatrice était alors invitée à décrire chacune des stratégies utilisées ainsi que son contexte d'utilisation. Au temps 2, les questions suivantes ont été posées: (1) «Quels moyens concrets utilisez-vous actuellement pour faire face aux comportements de (prénom de l'enfant)?» et (2) «Considérezvous qu'il y a eu un changement dans vos stratégies éducatives suite à l'intervention de SAEM?». Dans les cas où l'enfant avait quitté le milieu de garde ou avait changé de groupe au temps 2, seule la seconde question était posée. Cette seconde collecte de données a été réalisée dans le cadre d'un entretien téléphonique. Même si ce choix impliquait l'utilisation d'une procédure différente de celle du temps 1 , il a été retenu parce qu'il était moins exigeant pour les répondantes en terme de disponibilité, ce qui assurait un plus haut taux de réponse. En outre, comme cet entretien était réalisé par la même personne que l'entrevue du temps 1, la communication était facilitée, même si elle se déroulait au téléphone, par le fait qu'il y avait déjà un contact d'établi avec les répondantes.

Lors de cet entretien, les éducatrices ont également été questionnées sur les éléments ayant facilité et les éléments ayant fait obstacle à l'intervention, et elles ont été invitées à fournir une appréciation du programme SAEM. Cette mesure d'appréciation a été réalisée avec la version abrégée de l'échelle de satisfaction [Client Satisfaction Inventory/Short Form (CSI-SF)] développée par McMurtry et Hudson (2000). Il s'agit d'une échelle de type Likert à 7 points comportant 9 énoncés. Cet instrument présente une bonne validité de construit et une excellente cohérence interne (alpha de Cronbach de 0,89 dans la présente étude). Quelques données 
complémentaires, portant notamment sur les objectifs d'intervention et les modalités d'aide, ont été recueillies dans les dossiers cliniques des enfants. Trente-cinq éducatrices ont participé à cette deuxième entrevue, les autres n'étant pas disponibles pour diverses raisons (refus, changement d'emploi). En moyenne, le temps écoulé entre les deux entrevues est de 4,5 mois, avec un minimum de trois mois et un maximum de sept mois et demi.

Le contenu des entrevues a fait l'objet d'un enregistrement audio et d'une transcription intégrale. Il a ensuite été analysé selon les procédures habituelles de l'analyse de contenu constitué : préparation du matériel, préanalyse, exploitation et analyse et interprétation des résultats (Deslauriers et Mayer, 2000). Un système de catégorisation mixte a été privilégié; une partie des catégories est dérivée de la typologie de Essa (2002) et l'autre partie a été induite en cours d'analyse. Le matériel a été analysé à l'aide du logiciel NVivo 1.0.

\section{Profil des répondantes}

Les éducatrices qui ont participé à l'étude proviennent de trois milieux: Centre de la petite enfance (CPE) en installation (61\% des répondantes), CPE en milieu familial $(24,4 \%)$ et milieu de garde privé $(14,6 \%)$. Ce sont des femmes dont l'âge varie de 22 à 61 ans, avec une moyenne de 33,3 ans $(E T=8,9)$. La majorité d'entre elles $(77,5 \%$ ) ont un diplôme d'études collégiales. Leur expérience en milieu de garde se situe en moyenne à 8 ans. Plus précisément, elle est de moins de 2 ans pour 17,1\%, de 2 à 5 ans pour $31,7 \%$, de 6 à 10 ans pour $22 \%$ et de plus de 10 ans pour 29,3\%. Presque toutes ces personnes $(95,1 \%$ ) travaillent à temps plein (4 ou 5 jours) et elles s'occupent de groupes d'enfants dont la taille varie de 5 à 11 enfants (moyenne: 8,2, $E T=1,6)$.

\section{Les enfants visés par le programme}

La demande de service adressée au Programme SAEM par ces éducatrices porte sur 41 enfants, majoritairement (78\%) des garçons, âgés de 2 à 5 ans, pour un âge moyen de 3,3 ans. Une forte proportion des enfants de l'échantillon vivent au sein d'une famille biparentale intacte ( $80 \%)$, c'est-à-dire en compagnie de leur père et de leur mère. Parmi les huit enfants ne vivant plus en famille intacte, six vivent le plus souvent avec leur mère et deux vivent en garde partagée.

Le tableau 1 fournit la liste des besoins, considérés sous-jacents à leurs problèmes de comportement, qui sont mentionnés dans les plans d'intervention selon le nombre d'enfants qui en sont l'objet. Aux fins de la présentation, ces besoins ont été distingués en deux catégories selon qu'ils demandent une réaction de l'adulte ou qu'ils renvoient plutôt au comportement de l'enfant lui-même. 
Les stratégies éducatives du personnel en milieu de garde avec les enfants présentant des troubles du comportement: appréciation des effets d'un programme

Tableau 1 : Besoins des enfants tels qu'identifiés dans le plan d'intervention destiné au milieu de garde $(\mathrm{N}=35)$

\begin{tabular}{|c|c|c|}
\hline & Nombre d'enfants & Besoin identifié dans le plan d'intervention \\
\hline \multirow{8}{*}{$\begin{array}{l}\text { Besoin qui exige } \\
\text { une réaction de l'adulte }\end{array}$} & 8 & Etre encadré, avoir des limites \\
\hline & 4 & Être stimulé, motivé sur différents plans \\
\hline & 4 & Améliorer son estime de soi \\
\hline & 3 & Être arrêté \\
\hline & 3 & Sécurité \\
\hline & 2 & Cohérence entre la maison et le service de garde \\
\hline & 1 & Distanciation par rapport à l'adulte \\
\hline & 1 & Pouvoir \\
\hline \multirow{7}{*}{$\begin{array}{r}\text { Besoin qui renvoie } \\
\text { au comportement } \\
\text { de l'enfant }\end{array}$} & 7 & Apprendre à s'exprimer ou à s’affirmer de façon adéquate \\
\hline & 3 & Apprendre à s'approprier une place confortable, positive dans le groupe \\
\hline & 3 & Apprendre à gérer son agressivité \\
\hline & 2 & Apprendre à respecter les consignes \\
\hline & 2 & Apprendre à respecter ses limites \\
\hline & 1 & Dépenser son plein d'énergie \\
\hline & 1 & Développer son attention \\
\hline
\end{tabular}

\section{Les interventions}

Le nombre moyen d'interventions directes réalisées par l'intervenante SAEM est de 7,5 par enfant $(E T=3,7)$. Une intervention directe correspond à « une action concrète auprès de l'enfant, de l'éducatrice ou des parents. L'intervention directe ne constitue pas par exemple une simple prise de rendez-vous par téléphone». Le nombre moyen d'interventions réalisées en milieu de garde (moyenne : 5,1;ET=2,6) est plus élevé que le nombre d'interventions en milieu familial (moyenne: 3,4; $E T=2,1$ ). Précisons à cet égard que dans le cas de 8 enfants, il n'y a aucune intervention en milieu familial; ils ne sont pas comptabilisés dans la moyenne.

\section{La nature des stratégies éducatives}

L'analyse des propos recueillis auprès des éducatrices a conduit à distinguer 12 stratégies éducatives (S1 à S12) qui peuvent être regroupées en deux types: (1) celles qui sont utilisées en amont de la manifestation du comportement problématique et (2) celles qui constituent une réaction au comportement problématique. Les premières réfèrent à des actions posées avant que l'enfant manifeste un comportement problématique ou en dehors des moments où il se désorganise. Ces stratégies, qui visent à diminuer le risque que le comportement problématique se présente, sont: les actions préventives (interventions indirectes et promotion de comporte- 
ments positifs), le renforcement positif, l'utilisation de la dynamique du groupe, la stimulation de l'enfant et les interventions normalisantes. Ces stratégies s'actualisent à travers différents comportements qui sont illustrés dans le tableau 2.

Tableau 2 : Nature des comportements correspondant aux stratégies en amont du comportement

\begin{tabular}{|c|c|}
\hline Actions préventives & \\
\hline \multirow{2}{*}{ Interventions indirectes } & Organiser l'environnement physique \\
\hline & Ajuster les modalités de fonctionnement du groupe \\
\hline \multirow{8}{*}{$\begin{array}{l}\text { Promotion de } \\
\text { comportements positifs }\end{array}$} & Ajuster les exigences \\
\hline & Rappeler les consignes et les faire répéter par l'enfant \\
\hline & Formuler positivement les consignes \\
\hline & Annoncer des conséquences \\
\hline & Présenter des options possibles \\
\hline & Attribuer des responsabilités à l'enfant \\
\hline & Placer l'enfant à proximité d'une éducatrice \\
\hline & Éviter les situations à risque de problèmes de comportement \\
\hline \multirow{4}{*}{ Renforcement positif } & Féliciter et encourager verbalement et non verbalement \\
\hline & Utiliser un tableau de motivation \\
\hline & Attribuer des récompenses, privilèges \\
\hline & Mettre en valeur les forces de l'enfant \\
\hline \multirow{6}{*}{$\begin{array}{l}\text { Stratégies centrées sur la } \\
\text { dynamique du groupe }\end{array}$} & Sensibiliser les autres enfants à la situation \\
\hline & Outiller les autres enfants pour résoudre les situations conflictuelles \\
\hline & Solliciter la collaboration des autres enfants \\
\hline & Jumeler les enfants \\
\hline & Encourager la résolution de problèmes entre enfants \\
\hline & Changer l'enfant de groupe périodiquement \\
\hline \multirow{2}{*}{$\begin{array}{l}\text { Interventions } \\
\text { normalisantes }\end{array}$} & Diminuer les interventions publiques à l'endroit de l'enfant \\
\hline & Réagir plus discrètement \\
\hline \multirow{2}{*}{ Stimulation de l'enfant } & Stimuler les fonctions cognitives par des jeux appropriés \\
\hline & Proposer des activités pédagogiques \\
\hline
\end{tabular}

Les stratégies utilisées en réaction au comportement problématique réfèrent à des actions posées au moment où l'enfant présente un problème de comportement. Elles correspondent aux actions suivantes : la prise de conscience, l'application de conséquences, l'ignorance du comportement, le retrait volontaire, la réorientation, le défoulement de l'enfant et le contact physique avec l'enfant. Leur nature est précisée dans le tableau 3. 
Les stratégies éducatives du personnel en milieu de garde avec les enfants présentant des troubles du comportement: appréciation des effets d'un programme

Tableau 3 : Nature des stratégies utilisées en réaction au comportement

\begin{tabular}{|c|c|}
\hline Prise de conscience & $\begin{array}{l}\text { Discuter de la situation } \\
\text { Faire une démonstration de la situation (l'imiter) }\end{array}$ \\
\hline \multirow{4}{*}{$\begin{array}{l}\text { Application } \\
\text { des conséquences }\end{array}$} & Mettre un terme à une activité \\
\hline & Enlever un privilège \\
\hline & Exiger réparation \\
\hline & Retirer du groupe \\
\hline \multirow{2}{*}{$\begin{array}{l}\text { Ignorance } \\
\text { du comportement }\end{array}$} & Démontrer une ignorance intentionnelle \\
\hline & Faire preuve de tolérance \\
\hline Retrait volontaire & Offrir à l'enfant de s'isoler du groupe \\
\hline \multirow{2}{*}{ Réorientation } & Attirer l'attention de l'enfant sur autre chose \\
\hline & Faire appel à la contribution de l'enfant \\
\hline \multirow{2}{*}{ Défoulement } & Permettre à l'enfant d'extérioriser ses sentiments \\
\hline & Faire des activités qui permettent de bouger \\
\hline \multirow{3}{*}{$\begin{array}{l}\text { Contact physique } \\
\text { avec l'enfant }\end{array}$} & Maintenir une proximité physique avec l'enfant \\
\hline & Se mettre à la hauteur de l'enfant pour lui parler \\
\hline & Lui toucher pour le rassurer \\
\hline
\end{tabular}

Le tableau 4 trace un portrait de l'utilisation de ces différentes stratégies éducatives au temps 1. L'examen des résultats, sans distinction du type de milieux de garde, indique que cinq stratégies sont utilisées par plus de $50 \%$ des éducatrices: les actions préventives, le renforcement positif, l'utilisation de la dynamique du groupe, l'application de conséquences et la prise de conscience. En contrepartie, certaines stratégies sont utilisées par moins de $10 \%$ des éducatrices, soit les interventions normalisantes et le retrait volontaire.

Même si cet exercice doit être considéré avec prudence compte tenu du faible nombre de répondantes, la comparaison des stratégies selon le type de milieux de garde fait ressortir quelques observations intéressantes. Ainsi, quatre des cinq stratégies utilisées par une majorité d'éducatrices sont partout les mêmes, soit les actions préventives, le renforcement positif, l'application de conséquences et la prise de conscience. Seules les stratégies centrées sur la dynamique du groupe dérogent à cette constante en étant utilisées par plus de $50 \%$ des éducatrices en installation et en milieu de garde privé, mais par $30 \%$ des éducatrices en milieu familial. On note également que la prise de conscience est utilisée par environ $50 \%$ des éducatrices en milieu de garde privé et en installation alors qu'elle est mise de l'avant par $90 \%$ des éducatrices en milieu familial. On note également un écart dans l'utilisation du renforcement positif entre les éducatrices en installation (64\%) et celles en milieu de garde privé (100\%). Parmi les stratégies moins répandues, le défoulement de l'enfant n'est pas utilisé par les éducatrices en milieu familial alors qu'il l'est par le tiers des éducatrices en milieu de garde privé. En outre, les données indiquent l'absence de 
quelques stratégies dans certains types de milieux de garde. Ainsi, le retrait volontaire et le défoulement de l'enfant ne sont pas utilisés par les éducatrices en milieu familial alors que les actions normalisantes sont absentes en milieu familial et en garderie privée.

Tableau 4 : Portrait des stratégies éducatives utilisées en Temps 1 en fonction du type de milieux de garde de l'éducatrice

\begin{tabular}{|c|c|c|c|c|c|c|c|c|}
\hline \multirow{2}{*}{$\begin{array}{l}\text { Stratégies éducatives } \\
\begin{array}{l}\text { Stratégies en amont des } \\
\text { manifestations du comportement } \\
\text { problématique }\end{array}\end{array}$} & \multicolumn{2}{|c|}{$\begin{array}{c}\text { Utilisation par } \\
\text { I'ensemble } \\
\text { des } \\
\text { éducatrices } \\
(\mathbf{N}=41)\end{array}$} & \multicolumn{2}{|c|}{$\begin{array}{c}\text { Utilisation par } \\
\text { les éducatrices } \\
\text { en CPE } \\
\text { installation } \\
(\mathrm{N}=\mathbf{2 5}) \\
\end{array}$} & \multicolumn{2}{|c|}{$\begin{array}{l}\text { Utilisation par } \\
\text { les éducatrices } \\
\text { en CPE } \\
\text { Mil. familial } \\
(\mathrm{N}=10)\end{array}$} & \multicolumn{2}{|c|}{$\begin{array}{l}\text { Utilisation par } \\
\text { les éducatrices } \\
\text { en milieu } \\
\text { privé } \\
(\mathrm{N}=6)\end{array}$} \\
\hline & $\mathrm{N}$ & $\%$ & $\mathrm{~N}$ & $\%$ & $\mathrm{~N}$ & $\%$ & $N$ & $\%$ \\
\hline S 2- Renforcement positif & 30 & 73,2 & 16 & 64,0 & 8 & 80,0 & 6 & 100 \\
\hline S1 - Actions préventives & 27 & 65,9 & 16 & 64,0 & 7 & 70,0 & 4 & 66,8 \\
\hline \multirow{2}{*}{ 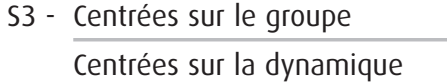 } & 21 & 51,2 & 15 & 60,0 & 3 & 30,0 & 3 & 50,0 \\
\hline & 7 & 17,1 & 5 & 20,0 & 1 & 10,0 & 1 & 16,7 \\
\hline S4- Interventions normalisantes & 2 & 4,9 & 2 & 8,0 & - & - & - & - \\
\hline $\begin{array}{l}\text { Stratégies en réaction au } \\
\text { comportement problématique }\end{array}$ & $\mathrm{N}$ & $\%$ & $\mathrm{~N}$ & $\%$ & $\mathrm{~N}$ & $\%$ & $\mathrm{~N}$ & $\%$ \\
\hline S7 - Application de conséquences & 36 & 87,8 & 20 & 80,0 & 10 & 100 & 6 & 100 \\
\hline S6 - Prise de conscience & 25 & 61,0 & 13 & 52,0 & 9 & 90,0 & 3 & 50,0 \\
\hline S8 - Ignorance du comportement & 12 & 29,3 & 7 & 28,0 & 4 & 40,0 & 1 & 16,7 \\
\hline $\begin{array}{l}\text { S12 - Contact physique avec } \\
\text { l'enfant }\end{array}$ & 12 & 29,3 & 7 & 8,0 & 3 & 0 & 2 & 3,3 \\
\hline S10 - Réorientation & 11 & 26,8 & 8 & 32,0 & 2 & 20,0 & 1 & 16,7 \\
\hline S11 - Défoulement de l'enfant & 7 & 17,1 & 5 & 20,0 & - & - & 2 & 33,3 \\
\hline S9 - Retrait volontaire & 4 & 9,8 & 3 & 12,0 & - & - & 1 & 16,7 \\
\hline
\end{tabular}

Le tableau 5 compare l'utilisation de chacune des stratégies avant et après le programme SAEM. Cette comparaison porte seulement sur 25 éducatrices, soit celles qui étaient toujours responsables de l'enfant à l'origine de la demande de service, au temps 2. Les données indiquent que sept stratégies sont utilisées par moins d'éducatrices à la fin de l'intervention. La diminution du nombre d'éducatrices utilisant ces stratégies varie d'une à huit. En ordre décroissant, ces stratégies sont: les stratégies centrées sur le groupe et sa dynamique (-8), l'application de conséquences (-6), les actions préventives (-5), l'ignorance du comportement (-4), le renforcement positif (-2), la réorientation (-2) et les interventions normalisantes (-1). D'autre part, quatre stratégies sont utilisées par plus d'éducatrices au temps 2, mais l'augmentation est 
Les stratégies éducatives du personnel en milieu de garde avec les enfants présentant des troubles du comportement: appréciation des effets d'un programme

faible. Il s'agit du contact physique avec l'enfant et du défoulement de l'enfant $(+2)$, ainsi que la stimulation de l'enfant et la prise de conscience $(+1)$.

Tableau 5 : Comparaison entre l'utilisation des stratégies éducatives au I1 et au T2

\begin{tabular}{|c|c|c|c|c|c|c|}
\hline \multirow{2}{*}{$\begin{array}{l}\text { Stratégies éducatives } \\
\text { Stratégies en amont des manifestations } \\
\text { du comportement problématique }\end{array}$} & \multicolumn{2}{|c|}{$\begin{array}{l}\text { Utilisation } \\
\text { par les } \\
\text { éducatrices } \\
\text { au T1 } \\
(\mathrm{N}=\mathbf{2 5})\end{array}$} & \multicolumn{2}{|c|}{$\begin{array}{l}\text { Utilisation } \\
\text { par les } \\
\text { éducatrices } \\
\text { au T2 } \\
(\mathrm{N}=\mathbf{2 5})\end{array}$} & \multicolumn{2}{|c|}{$\begin{array}{c}\text { Écart } \\
\text { dans les } \\
\text { utilisations } \\
(\mathbf{N}=25)\end{array}$} \\
\hline & $\mathrm{N}$ & $\%$ & $\mathrm{~N}$ & $\%$ & $\mathrm{~N}$ & $\%$ \\
\hline S2 - Renforcement positif & 18 & 72,0 & 16 & 64,0 & -2 & $-8,0$ \\
\hline S1 - Actions préventives & 17 & 68,0 & 12 & 48,0 & +5 & $-20,0$ \\
\hline \multirow{2}{*}{ 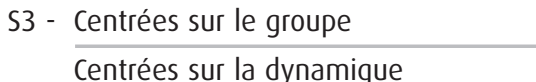 } & 16 & 64,0 & 8 & 32,0 & -8 & $-32,0$ \\
\hline & 6 & 24,0 & 7 & 28,0 & +1 & $+4,0$ \\
\hline S4 - Interventions normalisantes & 2 & 8,0 & 1 & 4,0 & -1 & $-4,0$ \\
\hline $\begin{array}{l}\text { Stratégies en réaction au comportement } \\
\text { problématique }\end{array}$ & $\mathrm{N}$ & $\%$ & $\mathrm{~N}$ & $\%$ & $\mathrm{~N}$ & $\%$ \\
\hline S7 - Application de conséquences & 21 & 84,0 & 15 & 60,0 & -6 & $-24,0$ \\
\hline S6 - Prise de conscience & 15 & 60,0 & 16 & 64,0 & +1 & $+4,0$ \\
\hline S8 - Ignorance du comportement & 7 & 28,0 & 3 & 12,0 & -4 & $-16,0$ \\
\hline S12 - Contact physique avec l'enfant & 7 & 28,0 & 9 & 36,0 & +2 & $+8,0$ \\
\hline S10 -Réorientation & 4 & 16,0 & 2 & 8,0 & -2 & $-8,0$ \\
\hline S11 - Défoulement de l'enfant & 3 & 12,0 & 5 & 20,0 & +2 & $+8,0$ \\
\hline S9 - Retrait volontaire & 1 & 4,0 & 1 & 4,0 & 0 & 0,0 \\
\hline
\end{tabular}

Au temps 1, le nombre moyen de stratégies utilisées est de 4,60 $(E T=1,47)$ alors qu'il est de 3,80 (ET = 1,71) au temps 2. Pour 14 éducatrices (56\%), la diversité des stratégies utilisées diminue alors qu'elle augmente pour cinq seulement $(20 \%)$. Donc, on observe une tendance allant dans le sens d'une diminution de la variété des stratégies utilisées, mais il n'est pas impossible que cette tendance soit attribuable à la différence dans la procédure de collecte des données.

Bien qu'il soit difficile de statuer sur l'influence du programme sur ces changements dans les stratégies utilisées, selon l'appréciation des éducatrices, le programme conduit à des effets positifs. En effet, à la question : "Considérez-vous qu'il y a eu un changement dans vos stratégies éducatives suite à l'intervention de SAEM?», les trois quarts $(77$ \%) des répondantes considèrent que l'intervention de SAEM a contribué à modifier leurs stratégies éducatives. Comme l'illustre le tableau 6, ce changement prend différentes formes. Dans certains cas, elles recourent à des stratégies qu'elles utilisaient déjà, mais les appliquent différemment. Dans d'autres cas, elles indiquent avoir amorcé l'utilisation de stratégies qu'elles connaissaient, mais n'avaient jamais appliquées auparavant. Enfin, quelques situations donnent lieu à la mise en œuvre de stratégies qui étaient inconnues jusque-là. 
Tableau 6 : Illustration de chacun des niveaux de perception du changement à partir des propos des éducatrices

\section{L'intervention n'a apporté aucun changement}

Non, je pense que ça n'a pas fait de changement. Je trouvais que j'avais comme pas de piste d'intervention puis je passais mon temps à remplir des questionnaires. C'est ça qui machalait un petit peu. (Éduc.\#2)

Non, il n'y a pas vraiment eu de changement dans mes pratiques. Les interventions qui m'avaient été suggérées par (l'intervenant), ce sont des choses que déjà en garderie, comme éducatrice, on fait déjà ça. Les interventions proposées comme telles, on les fait déjà. (Éduc.\#33)

\section{Utilisation des mêmes stratégies validées par l'intervenant}

Je te dirais que SAEM est venu un peu confirmer les choses que je faisais déjà avec lui, que j'étais dans la bonne voie. (Éduc.\#13)

II y a eu du renforcement par rapport à ce que je faisais déjà. Les visites de (I'intervenant) ont permis de confirmer nos interventions je pense. Ça a validé ce que je faisais. (Éduc.\#27)

\section{Utilisation des mêmes stratégies en les appliquant différemment} (nouvelles modalités d'application)

II y a des choses que je faisais avant, mais pas nécessairement de la même façon.

(Éduc.\#19)

(L'intervenant) m’a aussi proposé d'utiliser ces stratégies (que j'utilisais déjà) dans d'autres situations où je n'avais pas l'habitude de les utiliser. (Éduc.\#9)

\section{Utilisation de stratégies connues, mais non utilisées au temps 1} (rappel de stratégies par l'intervenant)

Axer les commentaires sur le comportement et non sur la personne, c'est une intervention qu'on connaît déjà, mais de se le faire rappeler particulièrement pour tel jeune, c'est aidant, ça m'aide à porter plus attention. (Éduc.\#11)

Des fois, on va vers le chemin le plus court et puis on n'y repense pas d'utiliser ces points-là, mais en me les rappelant, là j'y pense plus. (Éduc.\#30)

\section{Utilisation de nouvelles stratégies}

II y a eu beaucoup de changements pour intervenir avec elle. II y avait l'adaptation de mon matériel pour elle. Quand je lui parlais, je mebaissais tout le temps pour être vraiment à son niveau puis j'attendais vraiment qu'elle me regarde avec ses yeux. Mes interventions, je ne les faisais plus devant le groupe, je ne disais plus son nom fort. Ça c'est tout du nouveau. (Éduc.\#21)

Les fiches, c'est vraiment nouveau puis c'est vraiment magique. Je pense qu'elle est visuelle puis de lui dire comment je me sentais, elle ne le comprenait pas. Le voir, c'est très différent pour elle. (Éduc.\#23)

Avec le plan d'intervention, ça a beaucoup changé. On mettait plus l'accent sur des choses comparativement à avant où il y a des choses que je faisais puis que c'était un peu une perte. Ce n'était pas le temps pour lui de faire ces acquisitions-là. Alors on s'est vraiment concentré sur autre chose. (Éduc.\#36)

En ce qui a trait à leur satisfaction au regard de l'intervention, sur une échelle pouvant varier théoriquement entre 0 et 100, le résultat moyen pour l'ensemble des répondantes est de 85,5 (ET=16,2). Si le fait que les éducatrices qui perçoivent un changement se disent plus satisfaites des services reçus $(91,5)$ que celles qui ne perçoivent pas de changement $(65,3)$ n'est pas étonnant en soi, il n'en indique pas moins que l'échelle de satisfaction laisse place à l'expression d'une position moins favorable à l'égard du programme $(\mathrm{Z}(\mathrm{N}=35)=-3,59, \mathrm{p}<, 001)$. 
Les stratégies éducatives du personnel en milieu de garde avec les enfants présentant des troubles du comportement: appréciation des effets d'un programme

Tableau 7 : Perception des éducatrices au regard des changements dans leurs stratégies éducatives et satisfaction au regard de l'intervention

\begin{tabular}{|l|c|}
\cline { 2 - 2 } \multicolumn{1}{c|}{} & Total \\
\hline Changement perçu & $\mathbf{2 7}$ \\
Satisfaction : résultat moyen & 91,50 \\
(écart-type) & $(10,31)$ \\
\hline Aucun changement perçu & $\mathbf{8}$ \\
Satisfaction : résultat moyen & 65,28 \\
(écart-type) & $(16,44)$ \\
\hline TOTAL & $\mathbf{3 5}$ \\
& 85,50 \\
\hline
\end{tabular}

$Z=-3,59, p<0,001$ (test de Mann-Whitney)

Entre autres éléments sous-jacents à la satisfaction des éducatrices, il y a la possibilité de donner un sens aux comportements de l'enfant, la concordance des moyens proposés avec les préoccupations professionnelles, la simplicité des actions suggérées et la possibilité de transférer les acquis à d'autres situations
Entre autres éléments sous-jacents à la satisfaction des éducatrices, il y a la possibilité de donner un sens aux comportements de l'enfant, la concordance des moyens proposés avec les préoccupations professionnelles, la simplicité des actions suggérées et la possibilité de transférer les acquis à d'autres situations; leur portée ne se limite pas à la situation spécifique de l'enfant en difficulté.

(L'intervenant) m'a fait comprendre pourquoi il avait ce comportement-là, puis en comprenant, il me semble que ça va mieux quand on sait pourquoi le comportement est là. (Éduc. \#36)

Ça collait beaucoup avec ma personnalité,, donc c'était facile pour moi de mettre en pratique les points qu'on avait dits. (L'intervenant) me demandait toujours qu'estce que moi je voulais, qu'est-ce que moi j'aimais, puis les consignes de mon local, puis comment moi je fonctionnais. (L'intervenant) ne me proposait pas des affaires qui ne collaient pas avec moi. (Éduc. \#21)

Ce sont des petits trucs qui sont simples et efficaces, qui fonctionnent bien. (L'intervenant) a été très à l'écoute et a pris le temps vraiment d'essayer de comprendre un peu ce que nous autres on pouvait vivre à la garderie. (Éduc. \#26)

C'est un point fort qu'ils m'ont apporté qui est très utile dans ma pratique de tous les jours. C'est que tu ne t'attends pas à un résultat, donc tu n'es pas déçue. On y va tranquillement dans ça, puis à un moment donné, ça va ressortir. (Éduc. \#29)

Parmi les obstacles mentionnés, on retrouve les présences parfois trop espacées de l'intervenante SAEM, l'absence d'interventions directes de sa part auprès de l'enfant et le caractère parfois irréaliste de certaines suggestions. Leurs propos qui suivent illustrent ces commentaires négatifs à l'endroit du programme.

J'aurais aimé être capable de voir (l'intervenant) plus souvent. Si c'était à recommencer, je lui demanderais peut-être des rendez-vous un petit peu plus fréquents. Je trouve quand même que c'est un long bout à passer entre les rencontres. (Éduc. \#12) 
Les stratégies éducatives du personnel en milieu de garde avec les enfants présentant des troubles du comportement: appréciation des effets d'un programme

C'est sûr que j'aurais aimé ça que (l'intervenant) vienne passer des périodes pour intervenir directement avec l'enfant. Parce que souvent, voir intervenir quelqu'un qui saisit mieux les concepts que de seulement te les faire expliquer. (Éduc. \#21)

C'est ce que je reproche, des fois on nous propose des choses comme si on avait juste un enfant, mais on en a dix, tous avec d'autres troubles à corriger. (Éduc. \#2)

Dans le bilan qu'elles font du programme, les éducatrices identifient trois types de conditions de réussite de l'intervention : a) la qualité de l'appui qui leur est offert par l'intervenant SAEM, b) la concertation entre les trois principaux acteurs engagés envers l'enfant soit les parents, l'intervenant SAEM et elle-même et c) la rapidité d'accès aux services offerts par le programme SAEM. Ces conditions sont illustrées par les propos rapportés dans le tableau suivant.

Tableau 8 : Perception des éducatrices sur les conditions de succès du programme

\begin{tabular}{|l|l|}
\hline $\begin{array}{l}\text { Qualité de l'appui offert } \\
\text { par I'intervenant SAEM }\end{array}$ & $\begin{array}{l}\text { L'écoute, quand (I'intervenant) m'a écoutée, je l'ai beaucoup aimé. Je trouve que } \\
\text { (l'intervenant) ne me jugeait pas. Tout a été très positif. Je l'ai trouvé vraiment humain, } \\
\text { ouvert et je ne me suis pas sentie jugée. Je ne me suis pas sentie incompétente. (Éduc.\#23) }\end{array}$ \\
\hline $\begin{array}{l}\text { Concertation entre les } \\
\text { principaux acteurs } \\
\text { engagés vers l'enfant }\end{array}$ & $\begin{array}{l}\text { (L'intervenant) avait beaucoup de relations aussi avec les parents, au niveau de la famille, } \\
\text { C'était de voir que je ne travaillais pas dans un seul sens. (L'intervenant) a pu faire l'inter- } \\
\text { médiaire, le pont entre les deux milieux. (Éduc.\#15) }\end{array}$ \\
\hline $\begin{array}{l}\text { Rapidité d'accès aux } \\
\text { services }\end{array}$ & $\begin{array}{l}\text { Quand on a besoin de (l'intervenant), il est toujours là. (L'intervenant) est quand même } \\
\text { assez présent. Ce n'est pas tellement long non plus avant que (l'intervenant) se déplace. } \\
\text { En gros, c'est pas mal ça, la disponibilité. (Éduc.\#34) }\end{array}$ \\
\hline
\end{tabular}

\section{Discussion et conclusion}

Cette étude a été réalisée dans l'optique de mieux cerner les stratégies éducatives utilisées par le personnel des milieux de garde par rapport aux enfants qui présentent des problèmes de comportement et d'évaluer l'influence d'un programme visant à soutenir leur action auprès de ces enfants. Les données indiquent que le personnel de garde fait appel à une grande variété de stratégies lorsqu'il s'agit de composer avec un enfant difficile. Ces stratégies ne sont pas que réactives; plusieurs sont mises en place avec la préoccupation de prévenir les problèmes avant qu'ils se manifestent. Les résultats traduisent également que la majorité des éducatrices ayant bénéficié du Programme SAEM perçoivent des changements dans leurs stratégies éducatives. Leurs propos suggèrent qu'en faisant une meilleure analyse des besoins que l'enfant exprime à travers ses comportements, elles sont davantage en mesure de trouver des stratégies efficaces et, a contrario, d'éviter l'utilisation de stratégies moins appropriées. Les propos des éducatrices traduisent également que le 
Les stratégies éducatives du personnel en milieu de garde avec les enfants présentant des troubles du comportement: appréciation des effets d'un programme

Les résultats de cette étude viennent appuyer, d'une part, l'importance d'augmenter la formation des éducatrices en matière de gestion des comportements difficiles et, d'autre part, la nécessité de leur fournir un soutien adéquat pour composer avec ces enfants dont la présence

a souvent un effet perturbateur sur le groupe.
Programme SAEM influence l'ensemble de leur pratique, et non seulement l'intervention spécifique auprès de l'enfant en difficulté. Cependant, elles demeurent convaincues qu'une intervention efficace auprès d'un enfant en difficulté exige une action concertée des principaux acteurs qui gravitent autour de l'enfant. À cet égard, l'orientation du programme SAEM, à l'effet d'impliquer conjointement les parents et le personnel du milieu de garde dans une action concertée auprès de l'enfant, apparaît comme étant une option incontournable.

Les résultats de cette étude viennent appuyer, d'une part, l'importance d'augmenter la formation des éducatrices en matière de gestion des comportements difficiles et, d'autre part, la nécessité de leur fournir un soutien adéquat pour composer avec ces enfants dont la présence a souvent un effet perturbateur sur le groupe. Ils supportent également l'importance de la communication parents-éducatrice dans l'aide aux enfants en difficulté, la qualité de cette communication étant généralement considérée comme la pierre angulaire d'un partenariat efficace entre la famille et le milieu de garde (Coutu et coll., 2003; Owen, Wade et Barffot, 2000).

Évidemment, ces résultats doivent être examinés à la lumière des limites de cette étude, limites qui tiennent notamment à la taille de l'échantillon, à l'absence de groupe de comparaison et à la nature subjective de l'information collectée. Les choix méthodologiques effectués n'en ont pas moins permis de recueillir une information riche sur les stratégies des éducatrices en garderie et sur les changements perçus au regard de leurs stratégies éducatives suite à la participation à un programme d'intervention visant la prévention des problèmes d'adaptation sociale par l'intervention en milieu de garde. Par ailleurs, même si un autre volet de cette recherche (Turcotte, Saint-Jacques, St-Amand, Beaudoin et Champoux, 2004) a fait ressortir que la participation au programme SAEM s'était accompagnée d'une amélioration des comportements des enfants, il faut poursuivre les travaux sur l'évaluation des programmes de soutien au personnel en milieu de garde.

\section{Références bibliographiques}

BASTIEN, N., PLANTE, L. et COTTE, P. (1995). 1,2,3 Go ! Une initiative d'aide à la petite enfance. Sherbrooke: Actes du colloque en santé publique sur la prévention de la négligence à l'endroit des enfants. Direction générale de la santé publique. MSSSQ. Québec: Gouvernement du Québec.

BOUCHARD, A., GIROUARD, N. et ST-AMAND, A. (2004). Service d'aide à l'enfant et son milieu. Rapport d'activités 2003-2004. Québec : CLSC-CHSLD BasseVille-Limoilou-Vanier.

CANTIN, D. et PARADIS, T. (1993). Service d'aide à l'enfant et son milieu [s.l.]; www.saem.ca. 
CAPUANO, F. (1995). L'atelier Fluppy. Document audio visuel et guide. Montréal : Centre de Psychoéducation du Québec.

COUTU, S. LAVIGUEUR, S. DUBEAU, D. et Tardif, G. (2003). Les Centres de la petite enfance. Vole Grade en milieu familial. Un lieu de prévention des problèmes de développement et d'adaptation sociale des enfants. Hull : Université du Québec en Outaouis, Département de psychoéducation et de psychologie.

COUTU, S., LAVIGUEUR, S., DUBEAU, D. et Harvey, C. (1999). Les services de garde en milieu familial : un lieu de prévention à découvrir. Revue canadienne de Psycho-éducation, 28, 187-198.

CPPRG (Conduct Problems Prevention Research Group). (1997). Initial impact of the Fast Track prevention trial for cnduct problems. Journal of Consulting and Clinical Psychology. 67, 631-647.

DESBIENS, N. (2000). La réputation sociale des élèves manifestant des difficultés de comportement à l'école primaire: effet d'un programme de promotion des habiletés sociales et de coopération en classe ordinaire. Thèse de Doctorat, Département d'études sur l'enseignement et l'apprentissage, Faculté des sciences de l'éducation. Québec: Université Laval.

DESLAURIERS, J.P. et KERESIT, M. (1997). Le devis de recherche qualitative. dans J. Poupart, J.P. Deslauriers, L. Groulx, A. Laperrière, R. Mayer et O. Pires (sous la direction de), La recherche qualitative: Enjeux épistémologiques et méthodologiques Montréal: Gaëtan Morin Éditeur, 85-112.

ESSA, E. (2002). À nous de jouer en services de garde éducatifs. Guide pratique pour résoudre les problèmes comportementaux des enfants d'âge préscolaire. Québec: Les Publications du Québec.

FORTIN, L. et BIGRAS, M. (1997). Les facteurs de risque et les programmes de prévention auprès d'enfants en troubles de comportement. Québec : Éditions Behaviora Inc.

GROSENICK, J.K. et coll. (1985). Program Inventory: National Needs Analysis Project (Fostering Quality Program Planning and Design in the Area of Services Emotional Disturbances). University of Missouri : Columbia.

HAMEL, M. (1995). Les services d'éducation et d'intervention pour la petite enfance. Dans N. Royer (sous la direction de), Éducation et intervention au préscolaire Montréal : Gaétan Morin Éditeur, 3-27.

INSTITUTE OF MEDECINE (1994). Reducing risks for mental disorders: Frontiers for preventive intervention research.

KAISER, B. et SKLAR RASMINSKY, J. (1999). Meeting the Challenge. Effective Strategies for Challenging Behaviours in Early Childhood Environments. A project of the Canadian Child Care Federation in partnership with the Canadian Association of Family Resource Programs, the Canadian Institute of Child Health, the Child Welfare League of Canada and Family Service Canada. 
KAZDIN, A. (1987). Treatment of antisocial behavior in children: Current status and future directions. Psychological Bulletin. 102, 187-203.

KRATZER, L. et HODGINS, S. (1997). Adult outcomes of child conduct problems: A cohort study. Journal of Abnormal Child Psychology. 25, 65-81.

LACOURSE, E. COTE, S., NAGIN, D.S., VITARO, F., BRENDGEN, M. et TREMBLAY, R.E. (2002). A longitudinal-experimental approach to testing theories of antisocial behavior development. Development and Psychopathology, 15, 183-197.

MARSHALL, C. et ROSSMAN, G.B. (1999). Designing Qualitative Research ( $3^{\mathrm{e}}$ édition). Thousand Oaks, CA: Sage.

MAYER, R. et DESLAURIERS, J.P. (2000). Quelques éléments d'analyse qualitative. dans R. Mayer, F. Ouellet, M.C. Saint-Jacques et D. Turcotte (sous la direction de). Méthodes de recherche pour l'intervention sociale (159-190). Montréal : Gaëtan Morin Éditeur

MCMURTRY, S.L. et HUDSON, W.W. (2000). The Client Satisfaction Inventory: Results of an Initial Validation Study. Research on Social Work Practice, 10 (5), 644-663.

MINISTÈRE DE LA FAMILLE ET DE L'ENFANCE (1998). Jouer, c'est magique: Programme favorisant le développement global des enfants (tome 2). Québec: Les Publications du Québec.

MINISTERE DE LA SANTE ET DES SERVICES SOCIAUX DU QUEBEC (MSSSQ) (1997). Nouvelles dispositions de la politique familiale. Québec: Gouvernement du Québec.

PATTON, M.Q. (1987). How to Use Qualitative Methods in Evaluation. Newbury Park, CA : Sage.

PICHE, C., ROY, B. et COUTURE, G. (1992). Le projet Apprenti-Sage : une expérience d'intervention précoce et à long terme auprès d'enfants à hauts risques psychosociaux. Apprentissage et Socialisation, 15, 145-158.

POLIQUIN-VERVILLE, H. et ROYER, É. (1992). École et comportement. Les troubles du comportement: État des connaissances et perspectives d'intervention. Québec: Gouvernement du Québec, Ministère de l'Éducation.

REESE, L.E., VERA, E.M., SIMON, T.M. et IKEDA, R.M. (2002). The role of families and caregivers as risk and protective factors in preventing youth violence. Clinical Child and Family Psychology Review, 3, 61-77.

TURCOTTE, D., SAINT-JACQUES, M.C., ST-AMAND, A, BEAUDOIN, A. et L. CHAMPOUX (2004). Service d'aide à l'enfant et à sa famille: Rapport d'évaluation. Québec: Université Laval, Centre de recherche JEFAR, 147 p.

VITARO, F. et GAGNON. C. (sous la direction de) (2000). Prévention des problèmes d'adaptation chez les enfants et les adolescents. Montréal: Presses de l'Université du Québec. 
VITARO, F., DOBKIN, P.L., GAGNON, C. et LEBLANC, M. (1994). Les problèmes d'adaptation psychosociale chez l'enfant et l'adolescent: présence, prévalence et déterminants. Québec : P.U.Q.

WEBSTER-STRATTON, C. et HERBERT, M. (1995). Troubled families. Troubled children. Toronto : John Wiley et Sons.

WEISSBERG, R.P. et GREENBERG, M.T. (1998). School and community competence - enhacement and prevention programs. Dans W. Damon, I.E. Siegel et K.A. Renninger (Eds), Handbook of Child Psychology and Child Psychology in Practice. Vol. 4 (877-954). New York: John Wiley and Sons.

WERNER, E.E. et SMITH, R.S. (1989). Vulnerable but invincible. A longitudinal study of resilient children and youth. New York: Adams, Bannister and Cox. 